development over the 10 wks before term equivalent age in preterm infants.

Methods 35 infants (GA: $27.1 \pm 0.7$; BW: $937 \pm 172$ ) without morphine, were monitored with EEG/aEEG. Three periods were selected at 20-24 h, 32-36 h, 44-48 h. Minimum amplitude, $\%$ of time

Results Increased SATrate was positively associated with deltaGMv, inner and outer surface (resp $\beta: 7.4, p: 0.001 ; \beta: 46.6$, $p: 0.002 ; \beta: 57.5, p: 0.001)$. Consistent with these findings, ISI was negatively associated with changes in GMv, inner and outer surface $(\beta:-3.4, p: 0.007 ; \beta:-17.8, p: 0.034 ; \beta:-27.7, p: 0.006)$. Min aEEG and $\%$ of time $<5 \mu \mathrm{V}$ were associated with inner and outer surface at 40 wks (respectively: $\beta: 46.2, p: 0.043 ; \beta: 53.0$, $p: 0.041$; and $\beta:-2.9, p: 0.025 ; \beta:-3.5, p: 0.019)$. No effect on thickness and gyrification was found.

Conclusions Early brain activity seems to be associated with cortical development suggesting that adequate brain activity in the early neuronal networks is necessary to lead to growth and development of neonatal cerebral cortical brain, measured by structural MRI.

\section{0-063 DATA QUALITY IN DIFFUSION TENSOR IMAGING STUDIES OF THE PRETERM BRAIN}

${ }^{1} \mathrm{~K}$ Pieterman, ${ }^{1} \mathrm{~A}$ Plaisier, ${ }^{1} \mathrm{P}$ Govaert, ${ }^{2} \mathrm{~A}$ Leemans, ${ }^{3} \mathrm{M}$ Lequin, ${ }^{1} \mathrm{~J}$ Dudink. ${ }^{1}$ Neonatology, Erasmus University Medical Center - Sophia Children's Hospital, Rotterdam, Netherlands; ${ }^{2}$ Image Sciences Institute, University Medical Center Utrecht, Utrecht, Netherlands; ${ }^{3}$ Radiology, ErasmusMC - Sophia Children's Hospital, Rotterdam, Netherlands

\subsection{6/archdischild-2014-307384.131}

Background and aims Quantitative measurement of brain maturation is increasingly performed in preterm infants using diffusion tensor imaging (DTI). To study white matter properly, reliability of underlying DTI data is of paramount importance, as acquisition and processing steps can substantially affect DTI analyses. We systematically reviewed literature to raise awareness regarding these matters.

Methods We systematically reviewed studies published between 1991 and September 2013, in which DTI scanning of preterm infants was performed within 28 days after term-equivalent age. Based on our inclusion criteria, 75 preterm DTI studies were considered relevant and further analysed. We primarily focused on use of dedicated neonatal equipment, DTI acquisition parameters and processing methodology.

Results There was wide variation among different studies in acquisition and processing methodology, and frequently incomplete reporting of these settings. $25.3 \%$ reported the use of dedicated neonatal equipment. Data quality assessment was not reported in $34.7 \%$. Correction for artefacts and exclusion of datasets was not reported in $45.3 \%$ respectively $30.7 \%$. Only $54.7 \%$ of the studies reported specific correction methods. Tensor estimation methodology was reported in $82.7 \%$. Fast but less accurate tensor calculation algorithms were applied more frequently than advanced algorithms.

Conclusion

DTI acquisition and processing settings are described incompletely in current literature, and vary considerably among different neonatal DTI research groups. In addition, described settings do frequently not meet the highest standards possible. Hence the premature population should be regarded as one of the most challenging groups to image using DTI, maximal awareness regarding these matters is a prerequisite.

\section{Neonatal Immunity}

\section{0-064 DEVELOPMENT AND MATURATION OF MAIT CELLS IN HUMAN NEONATES: RELATIONS WITH GESTATIONAL AGE AND MICROBIAL INFECTION}

${ }^{1} \mathrm{~V}$ Biran, ${ }^{2} \mathrm{G}$ Ben Youssef, ${ }^{2} \mathrm{M}$ Tourret, ${ }^{3} \mathrm{~V}$ Houdoin, ${ }^{4} \mathrm{O}$ Baud, ${ }^{2} \mathrm{~S}$ Caillat-Zucman. ${ }^{1}$ Neonatal Intensive Care Unit, Robert Debre Hospital, Paris, France; ${ }^{2}$ Immunology, Robert Debre Hospital, Paris, France; ${ }^{3}$ Pneumology, Robert Debre Hospital, Paris, France; ${ }^{4}$ Neonatal Intensive Care Unit, Robert Debre Hospital, Paris, France

\subsection{6/archdischild-2014-307384.132}

Newborns, in particular preterm neonates, suffer a high frequency of microbial infections. MAIT cells are innate-like T cells expressing a semi-invariant V $\alpha 7.2-\mathrm{J} \alpha 33$ TCR which recognises MR1-restricted, microbial-derived riboflavin (vitamin B2) metabolites unique to bacteria and yeast.

We studied 151 newborns admitted in the Neonatology Department at Robert Debré Hospital divided into four groups according to gestational age (group 1: 24-27 wks; group 2: 28-31 wks; group 3: 32-36 wks; group 4: $>37$ wks). The rate and kinetics of MAIT cell expansion and maturation were determined longitudinally at birth (day 0), day 3, day 30 and day 60. We performed multiparametric 10-colour flow cytometry analyses using combinations of antibodies to CD45, CD3, CD4, CD8, TCR Va7.2, CD161, CD45RA, TCR V $\alpha 24$ and TCR $\gamma \delta$ on $100 \mathrm{ml}$ residual whole blood (left over of blood count), allowing characterisation of MAIT cells in parallel with other non-conventional and conventional T cells.

Our results show that the frequency of MAIT at birth is low and significantly differs according to gestational age (median at D0 group 1: 0.21\%; group 2: 0.14\%; group 3: 0.12\%; group 4: 0.06\%).

Of note, this frequency remains relatively stable over the first 2 months of life. However, the phenotype of MAIT cell changes after birth with rapid maturation and increased proportion of CD8aa cells. Significant difference was observed between high preterm neonates with and without maternofetal infection. Analysis of MAIT cell frequency in 20 twin pairs showed it was very similar, suggesting that it might be controlled by a genetic and/or early environmental factor.

In conclusion, the frequency of MAIT cells at birth is inversely correlated with gestational age, and is correlated with the presence of maternofetal microbial infection in preterm neonates. Whether it may reflect the presence of microbial products in amniotic liquid, and/or differences in the gut microbiota immediately after birth is under investigation.

\section{0-065 MACROPHAGE MIGRATION INHIBITORY FACTOR BALANCES NEONATAL INNATE IMMUNE RESPONSES}

${ }^{1} \mathrm{~T}$ Roger, ${ }^{2} \mathrm{M}$ Weier, ${ }^{2} \mathrm{~A}$ Schneider, ${ }^{3} \mathrm{~F} c \mathrm{gj}$ Sweep, ${ }^{4} \mathrm{~J}$ Bernhagen, ${ }^{1} \mathrm{~T}$ Calandra, ${ }^{2} \mathrm{E}$ Giannoni. ${ }^{1}$ Infectious Diseases Service, Centre Hospitalier Universitaire Vaudois and University of Lausanne, Lausanne, Switzerland; ' 2 Service of Neonatology and Infectious Diseases Service, Centre Hospitalier Universitaire Vaudois and University of Lausanne, Lausanne, Switzerland; ${ }^{3}$ Department of Laboratory Medicine, Radboud University, Nijmegen, Netherlands; ${ }^{4}$ Institute of Biochemistry and Molecular Cell Biology, Aachen University, Aachen, Germany

10.1136/archdischild-2014-307384.133 
Background The vulnerability to infection observed in newborns is associated with a limited ability to mount efficient immune responses. Elevated circulating levels of anti-inflammatory mediators (adenosine, prostaglandins,...) reduce production of proinflammatory cytokines by newborn monocytes upon microbial challenge. We hypothesised that macrophage migration inhibitory factor (MIF), a pro-inflammatory cytokine constitutively expressed in blood and immune cells, counter-balances the antiinflammatory milieu of newborns.

Methods MIF plasma levels were measured by ELISA in 200 healthy subjects, from birth to adulthood. Cord blood monocytes from term newborns were transfected with MIF siRNA, or were incubated with the MIF antagonist ISO-1, recombinant MIF, adenosine and prostaglandin E2 (PGE2) and then stimulated with endotoxin, bacterial lipopeptide, Escherichia coli and Group B Streptococcus (GBS). Intracellular proteins and cell-culture supernatants were collected to quantify MAPK phosphorylation by Western blot and cytokines by ELISA/Luminex.

Results Circulating MIF levels were 10-fold higher in newborns than adults, and decreased during infancy. Newborn monocytes expressed high MIF levels, and released MIF upon stimulation with Escherichia coli and GBS. Inhibition of MIF expression with MIF siRNA or MIF activity with ISO-1 reduced 1.5-5.7fold microbial product-induced secretion of pro-inflammatory (TNF, IL-1 $\beta$, IL-6, IL-8, IL-12p40, IL-12p70, IL-23) and antiinflammatory (IL-10, IL-20, IL-27) cytokines and phosphorylation of p38 and ERK1/2 MAPKs. Recombinant MIF counterregulated adenosine and PGE2-mediated inhibition of TNF production in Escherichia coli-stimulated newborn monocytes.

Conclusions MIF expression is developmentally regulated, with strikingly elevated levels in newborns compared to adults. High MIF levels at birth may act to balance pro/anti-inflammatory immune responses in newborns.

\section{Neonatal Resuscitation}

\section{0-066 ADMISSION HYPOTHERMIA IN VERY PRETERM INFANTS IS ASSOCIATED WITH MORTALITY - RESULTS FROM THE EPICE COHORT}

${ }^{1} \mathrm{E}$ Wilson, ${ }^{2} \mathrm{RF}$ Maier, ${ }^{3} \mathrm{~B}$ Misselwitz, ${ }^{4} \mathrm{~J}$ Zeitlin, ${ }^{5}$ AK Bonamy. ${ }^{1}$ CLINTEC, Karolinska Institutet, Stockholm, Sweden; ${ }^{2}$ Pediatrics, Philipps University, Marburg, Germany; ${ }^{3}$ Quality Assurance Hesse, Institute of Quality Assurance Hesse, Eschborn, Germany; ${ }^{4}$ Obstetrical Perinatal and Pediatric Epidemiology Research Team Center for Epidemiology and Biostatistics, INSERM, Paris, France; ${ }^{5}$ Women's and Children's Health Clinical Epidemiology Unit Department of Medicine Solna, Karolinska Institutet, Stockholm, Sweden

\subsection{6/archdischild-2014-307384.134}

Background Strategies to prevent heat loss in the delivery room after very preterm birth have been proven effective in randomised controlled trials. Nevertheless, we hypothesise that hypothermia at admission to neonatal care is still common and contributes to mortality after very preterm birth.

Methods The EPICE cohort included all births between $22+0$ and $31+6$ weeks of gestation in 19 regions from 11 European countries in 2011-2012. We studied infants surviving to admission to neonatal care ( $\mathrm{n}=7577)$. The association between temperature at admission and in-hospital mortality was analysed using logistic regression. The final model adjusted for gestational age, small for gestational age (SGA), Apgarscore $<7$ at $5 \mathrm{~min}$, infant sex and region of birth.

Results Of 6639 infants with data on body temperature at admission, 1670 infants $(25 \%)$ were hypothermic $\left(<36.0^{\circ} \mathrm{C}\right)$; $6 \%$ had temperatures $<35^{\circ} \mathrm{C}, 7 \%$ between 35.0 and $35.4^{\circ}$ and $12 \%$ between 35.5 and $35.9^{\circ}$. Body temperature at admission was inversely related to mortality. The crude odds ratio (OR) (95\% confidence interval [CI]) for mortality was 5.81(4.277.92) when temperature was $<35^{\circ} \mathrm{C} ; 3.32(2.35-4.69)$ at $35.0-$ $35.4^{\circ}$; and $1.61(1.18-2.19)$ at $35.5-35.9^{\circ}$ compared to normothermic infants $\left(36.5-37.5^{\circ} \mathrm{C}\right)$. After adjustment, temperatures below $35.5^{\circ} \mathrm{C}$ remained significantly associated with mortality, $1.94(1.32-2.83)$ at $<35^{\circ} \mathrm{C}$ and $1.91(1.30-2.82)$ at $35-35.4^{\circ} \mathrm{C}$ compared to normothermic infants.

Conclusion Hypothermia after very preterm birth contributes to mortality in modern perinatal care settings in Europe. Further studies should investigate if evidence-based heat loss prevention strategies have been implemented.

\section{0-067 THE INFLUENCE OF LOW QUALITY PULSE OXIMETRY SIGNALS ON EVALUATING INFANTS AT BIRTH}

${ }^{1} \mathrm{IC}$ Narayen, ${ }^{2} \mathrm{M}$ Smit, ${ }^{3} \mathrm{EW}$ van Zwet, ${ }^{4} \mathrm{JA}$ Dawson, ${ }^{5} \mathrm{NA}$ Blom, ${ }^{1} \mathrm{AB}$ te Pas. ${ }^{1}$ Neonatology, Leiden University Medical Center, Leiden, Netherlands; ${ }^{2}$ Obstetrics, Leiden University Medical Center, Leiden, Netherlands; ${ }^{3}$ Medical Statistics, Leiden University Medical Center, Leiden, Netherlands; ${ }^{4}$ Neonatal Services, The Royal Women's Hospital, Melbourne, Australia; ${ }^{5}$ Pediatric Cardiology, Leiden University Medical Center, Leiden, Netherlands

\subsection{6/archdischild-2014-307384.135}

Objectives Reference ranges for pulse oximetry (PO) measurements of oxygen saturation $\left(\mathrm{SpO}_{2}\right)$ and heart rate (HR) in infants at birth were generated excluding measurements with system messages (SyM), such as 'Low Signal Quality' or 'Low perfusion'. However, in clinical practice SyM often occur and may not be noticed. To assess the usability of measurements obtained with SyM, we investigated the differences of $\mathrm{SpO}_{2}$ and $\mathrm{HR}$ between measurements with and without SyM and if reference ranges would change when including data with SyM.

Methods $\mathrm{HR}$ and $\mathrm{SpO}_{2}$ of uncompromised term infants were recorded using Masimo PO. Differences in mean and standard deviation (SD) between measurements with and without SyM were calculated and compared with permutation tests. Centile charts were computed with and without SyM.

Results PO values of 138 neonates were obtained, resulting in 28,477 data points. SyM occurred in $46 \%$ of measurements. Most SyM occurred in the 3rd and 4th minute after birth. Mean $\mathrm{SpO}_{2}$ with SyM was lower $(\mathrm{p}<0.001)$, SD was similar to data without SyM. SyM included, centile charts of $\mathrm{SpO}_{2}$ are approximately 3\% lower, but not more dispersed. Mean HR was lower $(\mathrm{p}<0.001)$ and more dispersed $(\mathrm{p}<0.001)$ when SyM occurred. SyM included centile charts of HR have increased variability and are lower.

Conclusion In term infants at birth SyM on PO occurred frequently. $\mathrm{SpO}_{2}$ measurements with $\mathrm{SyM}$ are reliable for monitoring an infant's clinical condition. However, HR measurements with SyM should be used with caution as they may underestimate an infant's HR. 